\title{
Lipophilicity Influences Drug Binding to $\alpha 1$-Acid Glycoprotein F1/S Variants But Not to the A Variant
}

\author{
Kazuhiko Hanada ${ }^{1}$
}

Published online: 23 June 2017

(c) The Author(s) 2017. This article is an open access publication

\begin{abstract}
Objective Human $\alpha 1$-acid glycoprotein has genetic variants, the F1, S, and A variants, which can be separated isoelectrophoretically. These variants show differences in their affinity of binding to several drugs. In this study, we investigated the factors determining drug binding to these $\alpha 1$-acid glycoprotein genetic variants using disopyramide, warfarin, and tamsulosin as marker compounds.

Methods Binding of the marker drugs to human $\alpha 1$-acid glycoprotein was determined by ultra-filtration in the presence or absence of various other drugs. For screening of the $\alpha 1$-acid glycoprotein variants to which the marker drugs became bound, the effects of various other drugs on their binding were studied. The binding data were analyzed using a competitive inhibition model and the relationship between the estimated dissociation constants and physicochemical properties, such as $\log P$, was also analyzed.

Results The binding of tamsulosin was significantly decreased by aprindine, carvedilol, erythromycin, thioridazine, and warfarin, but not by disopyramide. The dissociation constants of drugs bound to $\mathrm{F} 1 / \mathrm{S}$ variants were significantly correlated with their lipophilicity, but those for the A variant were not.

Conclusions We were able to develop a simple screening method for determining individual $\alpha 1$-acid glycoprotein
\end{abstract}

Electronic supplementary material The online version of this article (doi:10.1007/s40268-017-0193-9) contains supplementary material, which is available to authorized users.

Kazuhiko Hanada

hanada@my-pharm.ac.jp

1 Department of Pharmacometrics and Pharmacokinetics, Meiji Pharmaceutical University, 2-522-1 Noshio, Kiyose, Tokyo 204-8588, Japan variants to which drugs would bind. The binding of drugs to F1/S variants may be determined mainly by drug lipophilicity.

\section{Key Points}

A simple screening method for determining individual $\alpha 1$-acid glycoprotein variants to which drugs would bind was developed.

The binding of drugs to F1/S variants may be determined mainly by drug lipophilicity.

\section{Introduction}

The binding of drugs to plasma protein is an important factor affecting drug pharmacokinetics. Alterations in the plasma protein binding of highly extracted drugs such as propofol after intravenous constant infusion cause a change in the unbound plasma drug concentration, but not the total concentration, at steady state [1]. However, alterations in the plasma protein binding of low-extracted (low-clearance) drugs such as warfarin and phenytoin, regardless of the route of administration, cause a change in the average total drug concentration, but not the unbound concentration, at steady state after repeated administration [2]. To predict changes in the unbound concentration of drugs in various clinical situations, the clinical significance of changes in protein binding should be investigated on the basis of drug clearance.

Most drugs bind mainly to albumin in plasma, but $\alpha_{1^{-}}$ acid glycoprotein (AGP) has been shown to play a role in 
the plasma binding of many weak base drugs [3]. $\boldsymbol{\alpha}_{1}$-Acid glycoprotein is also known to be an acute-phase reactant protein in acute inflammation such as that accompanying cancer and trauma [4-6], and the plasma concentration of AGP increases significantly as a result of upregulation of its specific messenger RNA [7]. Human AGP can be separated isoelectrophoretically into several variants, known as the F1, S, and A variants [8-10]. These variants are genetically determined by two different AGP genes, the $A G P-A$ gene encoding the variants ORM1 F1 and $\mathrm{S}$, and the $A G P-B / B^{\prime}$ gene encoding the variant ORM2 A $[8,9]$. The $A G P-A$ gene is similar to $A G P-B / B^{\prime}$ but has 22 significant base substitutions [8, 9]. Furthermore, the ORM1 $\mathrm{F} 1$ and $\mathrm{S}$ variants encoded by two alleles of $A G P-A$ differ in five amino acid residues.

Herve et al. investigated the binding of drugs to these separate AGP variants and showed that disopyramide and imipramine bind specifically to the A variant, warfarin to an F1/S variant mixture, and chlorpromazine and lignocaine to both variants $[11,12]$. These drugs exhibited uniform binding to AGP variants, suggesting that there is only one specific binding site for each drug on each variant. These previous studies have suggested that human AGP has two main genetic variants with one specific binding site. Recently, we reported that tamsulosin, an $\alpha 1$-adrenoceptor antagonist used for the treatment of benign prostatic hyperplasia, binds specifically to the F1/S variants of human AGP [13].

Direct determination of the binding characteristics of certain drugs is complex because of the difficulty in establishing individual analytical procedures and the influence of adsorption on membrane filter devices. Therefore, there has been a need to develop a simple procedure for identifying and quantifying the parameters of drug binding to human AGP variants. In this study, using disopyramide, warfarin, and tamsulosin as specific marker compounds, we investigated factors such as chemical structure and lipophilicity determining the selectivity of drug binding to AGP variants.

\section{Materials and Methods}

\subsection{Chemicals}

$\alpha_{1}$-Acid glycoprotein (Lot No. 125H9329), disopyramide phosphate, warfarin, miconazole, nicardipine hydrochloride, quinine, thioridazine hydrochloride, benazepril hydrochloride, erythromycin, quinidine hydrochloride, propafenone hydrochloride, felodipine, loperamide hydrochloride, and amitriptyline were obtained from Sigma Chemical Company (St. Louis, MO, USA). Tamsulosin hydrochloride and ${ }^{3} \mathrm{H}$-labeled tamsulosin were gifts from
Yamanouchi Pharmaceutical Co. (Tokyo, Japan). ${ }^{3} \mathrm{H}-\mathrm{la}-$ beled disopyramide was a gift from Nippon Roussel K.K. (Tokyo, Japan) and ${ }^{14} \mathrm{C}$-labeled warfarin was obtained from Amersham Biosciences Limited (Amersham, NJ, USA). Enantiomers of these drugs were separated by the enantioselective high-performance liquid chromatography (HPLC) method and their stereochemical purities were ascertained by stereoselective HPLC resolution, as reported previously [14]. Tamoxifen citrate and progesterone were obtained from Nakari Tesque (Kyoto, Japan). Glibenclamide and nitrendipine were obtained from Wako Pure Chemical Company (Tokyo, Japan). All other reagents used were of analytical grade.

\subsection{In-Vitro Protein-Binding Study}

The radio-labeled compounds were purified by HPLC as reported previously $[13,14]$, then dissolved in phosphate buffer and the solution was used on the same day. The binding of marker compounds to human AGP was determined by an ultrafiltration technique described previously $[13,14]$. The binding of $\left[{ }^{3} \mathrm{H}\right]$ tamsulosin was determined as follows: a $450-\mu \mathrm{L}$ aliquot of commercial AGP was added to $50 \mu \mathrm{L}$ of tamsulosin $(40,60,80,100,150,200,300$, or $500 \mu \mathrm{M})$ and $10 \mu \mathrm{L}$ of $\left[{ }^{3} \mathrm{H}\right]$ tamsulosin and $10 \mu \mathrm{L}$ of the 14 drugs tested $(9.62,19.2$, and $48.1 \mu \mathrm{M})$, and then the mixture was incubated for $15 \mathrm{~min}$ at $37{ }^{\circ} \mathrm{C}$. A $450-\mu \mathrm{L}$ aliquot of the mixture was poured onto a membrane filter (Ultrafree $^{\circledR}$ MC, MW cut-off 5000; Millipore, Bedford, MA, USA) and centrifuged at $2000 \times g$ for $5 \mathrm{~min}$ at $37^{\circ} \mathrm{C}$. The radioactivities of the mixture and the filtrate were determined using a liquid scintillation counter (LSC-700; Aloka, Tokyo, Japan). The bindings of $\left[{ }^{3} \mathrm{H}\right] S$-disopyramide and $\left[{ }^{3} \mathrm{H}\right] \mathrm{S}$-warfarin were determined by the same procedure as that described above, except for the membrane filter used (Ultrafree ${ }^{\circledR}-\mathrm{MC}, \mathrm{MW}$ cut-off 30,000 for disopyramide). Adsorption of these radio-labeled compounds to the membrane filters was negligible.

For screening of drugs binding extensively and specifically to AGP variants, the bindings of $\left[{ }^{3} \mathrm{H}\right] S$-disopyramide, $\left[{ }^{3} \mathrm{H}\right] S$-warfarin, and $\left[{ }^{3} \mathrm{H}\right]$ tamsulosin were determined in the absence and presence of the 14 compounds tested at a concentration 100-fold higher than that of the marker compounds (see Figs. 1, 2). When a drug was added at 100 -fold the concentration of a marker compound, we assumed that the total binding of the marker compound would be attributable to non-specific binding because specific binding would be inhibited completely under such conditions.

\subsection{Determination of Partition Coefficient}

The partition coefficient of each drug was determined as described previously [13]. Sörensen buffer and n-octanol 
Fig. 1 Effects of various drugs on the binding of $S$ -

disopyramide, $S$-warfarin, and tamsulosin to human $\alpha 1$-acid glycoprotein (only typical patterns are presented). a Effect of aprindine on the binding of $S$ dispyramide. b Effect of aprindine on the binding of $S$ warfarin. c Effect of simvastatin on the binding of tamsulosin [rhombus: control, square: with test drug $(9.62 \mu \mathrm{M})$, triangle: with test drug $(19.2 \mu \mathrm{M})$, circle: with test drug $(48.1 \mu \mathrm{M})$ ]

Fig. 2 Relationship between partition coefficients and dissociation constants
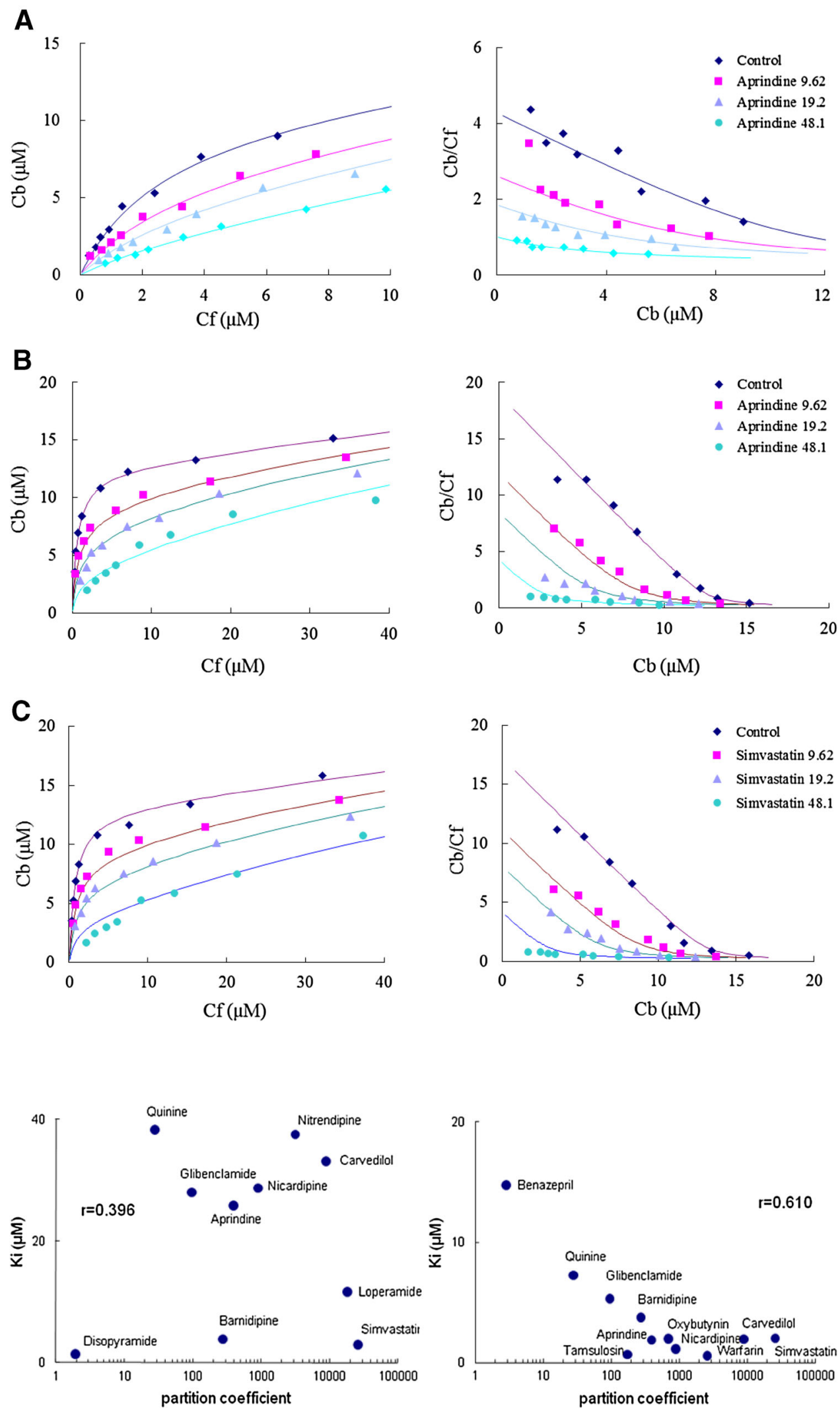
were used as the aqueous and organic phases, respectively. After equilibration, the radioactivities or concentrations in both the aqueous and organic phases were determined using a liquid scintillation counter and by HPLC, respectively.

\subsection{Data Analysis}

We assumed that non-specific binding of a drug (model compound) to AGP can be expressed as a constant, and not influenced by other drugs. The specific binding of a drug to AGP can be expressed under the assumption that the drug binds with uniform affinity, and shows competitive inhibition of the drug tested, based on a preliminary study. The drug-binding parameters were determined by non-linear least-squares regression using the computer program WinNonlin (Pharsight, Mountain View, CA, USA) following a competitive inhibition model:

$$
\begin{aligned}
& \mathrm{Cbs}=\frac{n \cdot \mathrm{AGP} \cdot S}{\mathrm{Kd}\left(1+\frac{I}{\mathrm{Ki}}\right)+S}+\alpha \cdot S \\
& \mathrm{Cbi}=\frac{n \cdot \mathrm{AGP} \cdot I}{\mathrm{Ki}\left(1+\frac{S}{\mathrm{Kd}}\right)+I}+\beta \cdot S,
\end{aligned}
$$

where $\mathrm{Cbs}, n$, AGP, $S, \mathrm{Kd}$, and $\alpha$ are the bound concentration of the substrate (model compound), AGP concentration, number of binding sites, unbound concentration of the substrate, dissociation constant of the substrate, and the non-specific binding constant of the substrate (for lowaffinity binding sites), respectively. $\mathrm{Cbi}, I, \mathrm{Ki}$, and $\beta$ are the bound concentration of the inhibitor (drug tested), unbound concentration of the inhibitor, dissociation constant of the inhibitor, and non-specific binding constant of the inhibitor, respectively. The linear relationship between the dissociation constants and partition coefficients of the drugs tested was analyzed using Pearson's correlation test with the computer program SPSS statistics 17.0 (IBM Japan, Ltd., Tokyo, Japan).

\section{Results}

Using a simple screening method, classification of the drugs binding to AGP variants was performed, and the binding affinity of various drugs was then determined using an indirect procedure. We investigated the factors determining the selectivity of drug binding to AGP variants, such as chemical structure and lipophilicity.

Fourteen drugs were studied for their binding to the A variant and an F1/S variant mixture of AGP. As a screening procedure for the binding, we investigated the inhibition of marker (radio-labeled) compound binding by adding the relevant drugs at a very high (100-fold) concentration. Inhibitors that reduced the specific binding of marker compounds by more than $70 \%$ were selected for subsequent quantitative analysis. The selected drugs were amitriptyline, aprindine, barnidipine, benazepril, carvedilol, diltiazem, doxazocin, erythromycin, glibenclamide, loperamide, miconazole, nicardipine, progesterone, propranolol, quinine, simvastatin, tamoxifen, and thioridazine. The binding of both disopyramide and warfarin was markedly inhibited by erythromycin, miconazole, nicardipine, propranolol, quinine, simvastatin, and thioridazine. Tamoxifen, amitriptyline, diltiazem, glibenclamide, and loperamide specifically inhibited the binding of $S$-disopyramide. Tamsulosin specifically inhibited the binding of warfarin.

All of the drugs listed in the Table in the Electronic Supplementary Material (ESM) competitively inhibited the binding of the marker compound, as judged from the Scatchard plot (typical data are shown in Fig. 1). The number of binding sites and nonspecific binding constants of the marker compounds were almost the same in the absence and presence of inhibitors (Fig. 1 and Table in the ESM). The binding affinity of thioridazine was comparable to that reported by others [11]. The binding affinity of aprindine, thioridazine, and quinine for the AGP A variant ( $S$-disopyramide binding site) was relatively high among the drugs tested. However, the binding affinity of nicardipine, thioridazine, simvastatin, and tamsulosin was relatively high for the AGP F1/S variant mixture ( $S$-warfarin binding site). The estimated inhibitory constants for tamsulosin were comparable to those obtained for $S$-warfarin (Table in the ESM).

The binding to each variant was then studied in relation to the dissociation constant and lipophilicity (Fig. 2). The dissociation constant estimated in this study was significantly correlated with $\log P$ for the F1/S variants but not for the A variant.

\section{Discussion}

In this study, we developed a simple screening method for determining the AGP variant for which certain drugs show binding affinity, and demonstrated that tamsulosin could be used as a new binding marker compound for specific binding to AGP F1/S variants. In the screening study, we selected various compounds, such as weakly acidic drugs, neutral drugs, and weakly basic drugs, which have highfraction binding values and/or are known to bind to AGP. It has been reported that tamoxifen, amitriptyline, imipramine, and thioridazine bind strongly to AGP $[11,12]$ and therefore these were used as positive controls. The results obtained using our simple screening method that tested the 
effect of an excess amount of inhibitor on the specific binding of marker drugs to AGP variants were comparable to the results obtained from quantitative analysis.

Tamsulosin binds extensively to human AGP and its plasma protein binding is altered in patients with renal failure [15] and acute inflammation [16]. Recently, we demonstrated that tamsulosin bound specifically to F1/S variants and that warfarin competitively inhibited this binding [13]. The dissociation constants of various drugs obtained using warfarin and tamsulosin were comparable, suggesting that tamsulosin was also a useful marker compound for studying the characteristics of drug binding to F1/S variants.

The dissociation constant was significantly correlated with the $\log \mathrm{P}$ for $\mathrm{F} 1 / \mathrm{S}$ variants, indicating that lipophilicity is an important factor for drug binding to AGP F1/S variants. Herve et al. have suggested that the binding site of F1/S variants is a large hydrophobic area with no obvious structural requirements for binding, and have pointed out that there is an insufficient number of highaffinity ligands for three-dimensional quantitative structure-activity analysis using comparative molecular field analysis [11]. The drugs used in this study that showed high affinity for F1/S variants have electron-attracting groups attached to the aromatic rings in their molecular structure (Figure in the ESM). Although drugs with high electronegativity showed more high-affinity binding, the steric hindrance around the electron-attracting group may conversely inhibit the binding of the drugs to $\mathrm{F} 1 / \mathrm{S}$ variants.

A study using three-dimensional quantitative structureactivity analysis has revealed some common structural features of A variant-binding drugs, including two rings present in high-affinity tricyclic drugs such as imipramine and $N$-methylated amines [11]. The $-N-\left(\mathrm{CH}_{2}\right) 3-N-$ functional group is present in the structures of thioridazine and aprindine, which showed high affinity for the A variant, and the distance of their two nitrogen atoms is similar; disopyramide has also two nitrogen atoms (Figure in the ESM). These results suggest that the binding of drugs to the A variant is partly determined by their lipophilicity field and tertiary ammonium functional group. However, we have previously reported enantioselective AGP binding of disopyramide, propranolol, and verapamil [10], and therefore, other determining factors may exist. Further investigations are required to clarify the characteristics of drug binding to AGP variants. When considering the relationship between the unbound fraction of a drug and AGP variants, it is necessary to bear in mind both the specific binding to genetic variants and the extent of nonspecific binding to both variants, as the unbound fraction is determined by binding to both highand low-affinity sites.

\section{Conclusion}

We have developed a simple screening method for determining the AGP variant to which drugs bind, and demonstrated that tamsulosin is a useful marker compound for specific binding to AGP F1/S variants. Binding to the A variant is determined partly by the drug's lipophilicity field and tertiary ammonium functional group. The binding of drugs to F1/S variants may be determined mainly by lipophilicity.

Acknowledgements The authors thank Nagisa Yamamoto and Kanako Fukuda for their excellent assistance in determining drug concentrations.

\section{Compliance with Ethical Standards}

Funding No funding was received for the preparation of this manuscript.

Conflict of interest Kazuhiko Hanada has no conflict of interest directly relevant to the contents of this article.

Open Access This article is distributed under the terms of the Creative Commons Attribution-NonCommercial 4.0 International License (http://creativecommons.org/licenses/by-nc/4.0/), which permits any noncommercial use, distribution, and reproduction in any medium, provided you give appropriate credit to the original author(s) and the source, provide a link to the Creative Commons license, and indicate if changes were made.

\section{References}

1. Hiraoka H, Yamamoto K, Okano N, Morita T, Goto F, Horiuchi R. Changes in drug plasma concentrations of an extensively bound and highly extracted drug, propofol, in response to altered plasma binding. Clin Pharmacol Ther. 2004;75:324-30.

2. Benet LZ, Hoener BA. Changes in plasma protein binding have little clinical relevance. Clin Pharmacol Ther. 2002;71:115-21.

3. Israili ZH, Dayton PG. Human alpha-1-glycoprotein and its interactions with drugs. Drug Metab Rev. 2001;33:161-235.

4. Daemen MARC, Heemskerk VH, vant's eer C, Denecker G, Wolfs TGAM, Vandenabeele P, Buurman WA. Functional protection by acute phase proteins alpha1-acid glycoprotein and alpha1-antitrypsin against ischemia/reperfusion injury by preventing apoptosis and inflammation. Circulation. 2000;102:1420-6.

5. Fournier T, Mejdoubi N, Monnet D, Durand G, Porquet D. Phenobarbital induction of alpha1-acid glycoprotein in primary rat hepatocyte cultures. Hepatology. 1994;20:1584-8.

6. Hanada K, Yamanaka E, Yamamoto N, Minami H, Kawai S, Sasaki Y, Ogata H. Effects of surgery and chronic disease states on the concentrations and phenotype distribution of $\alpha 1$-acid glycoprotein: studies in patients with breast cancer and patients with chronic inflammatory disease. Int $\mathrm{J}$ Clin Pharmacol Ther. 2011;49:415-21.

7. Duché JC, Urien S, Simon N, Malaurie E, Monnet I, Barré J. Expression of the genetic variants of human alpha-1-acid glycoprotein in cancer. Clin Biochem. 2000;33:197-202. 
8. Baumann P, Eap CB, Muller WE, Tillement JP. Alpha1-acid glycoprotein: genetics, biochemistry, physiological functions and pharmacology. New York: Alan R. Liss; 1989.

9. Dente L, Pizza MG, Metspalu A, Cortese R. Structure and expression of the genes coding for human alpha1-acid glycoprotein. EMBO J. 1987;6:2289-96.

10. Herve F, Duche JC, Barre J, Millot MC, Tillement JP. pH titration curves of the desialylated human alpha1-acid glycoprotein variants by combined isoelectrofocusing-electrophoresis: utilization in the development of a fractionation method for the protein variants by chromatography on immobilized metal affinity adsorbent. J Chromatogr. 1992;577:43-59.

11. Herve F, Duche JC, d'Athis P, Marche C, Barre J, Tillement JP. Binding of disopyramide, methadone, dipyridamole, chlorpromazine, lignocaine and progesterone to the two main genetic variants of human alpha1-acid glycoprotein: evidence for drugbinding differences between the variants and for the presence of two separate drug-binding sites on alpha1-acid glycoprotein. Pharmacogenetics. 1996;6:403-15.
12. Herve F, Caron G, Duche J-C, Gaillard P, Rahman NA, TsantiliKakoulidou A, Carrupt P-A, D'Athis P, Tillement J-P, Testa B. Ligand specificity of the genetic variants of human alpha1-acid glycoprotein: generation of a three-dimensional quantitative structure-activity relationship model for drug binding to the A variant. Mol Pharmacol. 1998;54:129-38.

13. Hanada K, Ohta T, Hirai M, Arai M, Ogata H. Enantioselective binding of propranolol, disopyramide, and verapamil to human alpha(1)-acid glycoprotein. J Pharm Sci. 2000;89:751-7.

14. Hanada K, Tochikura N, Ogata H. Selective binding of tamsulosin to genetic variants of human alpha1-acid glycoprotein. Biol Pharm Bull. 2007;30:1593-5.

15. Matsushima H, Kamimura H, Soeishi $Y$, Watanabe T, Higuchi S, Miyazaki M. Plasma protein binding of tamsulosin hydrochloride in renal disease: role of alpha1-acid glycoprotein and possibility of binding interactions. Eur J Clin Pharmacol. 1999;55:437-43.

16. Matsushima H, Watanabe $\mathrm{T}$, Higuchi S. Effect of alpha1-acid glycoprotein on the pharmacokinetics of tamsulosin in rats treated with turpentine oil. J Pharm Sci. 2000;89:490-8. 